\title{
Accuracy of Approximations to the Poynting Correction for Ice and Liquid Water
}

\begin{abstract}
Allan H. Harvey
National Institute of Standards and Technology, Boulder, CO 80305, USA

allan.harvey@nist.gov

Rigorous calculation of the Poynting correction, which describes the effect of pressure on the fugacity of a condensed phase, requires time-consuming evaluation of a thermodynamic potential such as the International Association for the Properties of Water and Steam (IAPWS-95) formulation for water. Simplifying approximations are used in many applications, but the error introduced by the approximations is seldom evaluated. In this work, first-order and second-order approximations were developed for the Poynting correction for both ice and liquid water (including supercooled liquid water), and their errors were evaluated by comparison to the full thermodynamic potentials. The range of conditions covered is from $-100{ }^{\circ} \mathrm{C}$ to $200{ }^{\circ} \mathrm{C}$ at pressures to $20 \mathrm{MPa}$. Some implications for the calculation of the enhancement factor used in humidity metrology are discussed.
\end{abstract}

Key words: fugacity; ice; Poynting correction; supercooled water; thermodynamics; water.

Accepted: October 24, 2017

Published: November 20, 2017

https://doi.org/10.6028/jres.122.041

\section{Introduction}

Many problems of practical and scientific interest involve equilibrium between a gas phase and a condensed phase that is either nearly pure liquid water or pure ice. Thermodynamic equilibrium entails equality of the fugacity (or, equivalently, the chemical potential) of water between the coexisting phases. The solution of the phase-equilibrium problem typically requires the fugacity of the pure condensed phase at the temperature $T$ and pressure $p$ of the system; we denote this by $f_{\mathrm{w}}^{\text {pure }}(T, p) . f_{\mathrm{w}}^{\text {pure }}(T, p)$ can be calculated if a thermodynamic potential is available that is valid at the conditions of interest, such as the International Association for the Properties of Water and Steam (IAPWS) standard for thermodynamic properties of fluid water [1, 2] or the IAPWS formulation for thermodynamic properties of ice Ih [3, 4].

In practice, calculating fugacity from such thermodynamic potentials is unwieldy, and for other substances in the condensed phase no such potential may be available. A convenient alternative is to start with the pure-component fugacity at the saturation pressure, $f_{\mathrm{w}}^{\text {pure }}\left(T, p^{\text {sat }}\right)$, which typically differs by only a small correction from the readily available pure-component vapor pressure $p^{\text {sat }}(T)\left[f_{\mathrm{w}}^{\text {pure }}\left(T, p^{\text {sat }}\right)=\varphi_{\mathrm{w}}^{\text {sat }} p^{\text {sat }}\right.$, where $\varphi_{\mathrm{w}}^{\text {sat }}\left(T, p^{\text {sat }}\right)$, the fugacity coefficient at saturation, is 1 for an ideal gas and typically slightly less than 1 for low and moderate pressures]. The key quantity then becomes the ratio of the fugacity at $(T, p)$ to that at saturation:

$$
\Pi=\frac{f_{\mathrm{w}}^{\text {pure }}(T, p)}{f_{\mathrm{w}}^{\text {pure }}\left(T, p^{\text {sat }}\right)},
$$

where $\Pi$ is the Poynting correction. The Poynting correction is rigorously related to an isothermal integral of the molar volume from $p^{\text {sat }}$ to $p$ : 


$$
\Pi(T, p)=\exp \left(\frac{1}{R T} \int_{p^{\mathrm{sat}}}^{p} v_{\mathrm{w}}(T, p) \mathrm{d} p\right)
$$

where $R$ is the molar gas constant and $v_{\mathrm{w}}$ is the molar volume of the condensed phase (water or ice in this work).

Equation (2) by itself does not provide much simplification, since it requires knowledge of the molar volume at all pressures from $p^{\text {sat }}$ to $p$. However, relatively simple approximations to Eq. (2) are widely used, such as assuming that $v_{\mathrm{w}}$ is independent of pressure [making the integral in Eq. (2) a simple product]. The errors introduced by these approximations are typically asserted to be small (at least for pressures that are not too high), but no systematic examination of their magnitude has been conducted.

Because of the existence of complete and quantitatively accurate thermodynamic potentials for liquid water $[1,2]$ and for ice [3, 4], it is now possible to provide quantitative evaluation of approximations to Eq. (2). In the following sections, we describe first-order and second-order approximations, and compare their performance against a rigorous calculation of the Poynting correction from the full thermodynamic potential. The comparisons are performed over a range of temperatures and pressures, including conditions where the condensed phase is metastable (supercooled) liquid water.

In addition, we will discuss the role of these approximations in the context of humidity metrology. It has recently been proposed $[5,6]$ to make use of fugacity-based measures for the traditional concept of relative humidity, which would provide more impetus for simple and accurate ways to calculate the fugacity of liquid water and ice.

\section{Development of Approximations}

\subsection{Thermodynamic Background}

A first-order approximation to the full Poynting correction is obtained by observing that liquid water and ice are nearly incompressible. This suggests that the molar volume $v_{\mathrm{w}}$ can be approximated throughout the range of integration in Eq. (2) by its value at saturation, $v_{\mathrm{w}}^{\text {sat }}$, producing a simple expression:

$$
\Pi_{1}(T, p)=\exp \left(\frac{v_{\mathrm{w}}^{\mathrm{sat}}(T)\left[p-p^{\mathrm{sat}}(T)\right]}{R T}\right)
$$

A second-order approximation is obtained by assuming that the change in molar volume with pressure can be described by a constant isothermal compressibility, $\kappa_{T}=\frac{-1}{v_{\mathrm{w}}}\left(\frac{\partial v_{\mathrm{w}}}{\partial p}\right)_{T}$, which is taken as its value at saturation, $\kappa_{T}^{\text {sat }}$. The resulting expression is

$$
\Pi_{2}(T, p)=\exp \left(\frac{v_{\mathrm{w}}^{\mathrm{sat}}(T)}{R T \kappa_{T}^{\mathrm{sat}}(T)}\left[1-\exp \left(-\kappa_{T}^{\mathrm{sat}}(T)\left[p-p^{\mathrm{sat}}(T)\right]\right)\right]\right)
$$

\subsection{Sources for Saturation Data}

The saturated molar volume $v_{\mathrm{w}}^{\mathrm{sat}}$ and the saturated isothermal compressibility $\kappa_{T}^{\text {sat }}$ could be obtained by computing derivatives of the full thermodynamic potential, but this would defeat the purpose of simplification. Simple, explicit correlations are needed for these quantities as a function of temperature.

For $v_{\mathrm{w}}^{\text {sat }}$ and $\kappa_{T}^{\text {sat }}$ of ice, we computed values from the IAPWS thermodynamic potential [3, 4]. The ratio of each quantity to its value at the triple point was fitted to a rational polynomial, leading to the following forms: 
and

$$
v_{\mathrm{w}}^{\mathrm{sat}, \mathrm{ce}}(T)=v_{\mathrm{w}, \mathrm{t}}^{\mathrm{sat}, \mathrm{ice}} \frac{1+\sum_{i=1}^{5} A_{i} \tau^{i}}{1+\sum_{j=1}^{3} B_{i} \tau^{i}},
$$

$$
\kappa_{T}^{\text {sat,ice }}(T)=\kappa_{T, \mathrm{t}}^{\mathrm{sat}, \mathrm{ice}} \frac{1+\sum_{i=1}^{5} C_{i} \tau^{i}}{1+\sum_{j=1}^{6} D_{i} \tau^{i}},
$$

where the temperature variable is defined by $\tau=1-T / T_{\mathrm{t}}$, and the triple-point temperature $T_{\mathrm{t}}$ is $273.16 \mathrm{~K}$. The values of the saturated molar volume and isothermal compressibility for ice at the triple point $[3,4]$ are $V_{\mathrm{w}, \mathrm{t}}^{\text {satice }}=1.96521015 \times 10^{-5} \mathrm{~m}^{3} / \mathrm{mol}$ and $\kappa_{T, \mathrm{t}}^{\text {satice }}=1.1779345 \times 10^{-10} \mathrm{~Pa}^{-1}$. Table 1 gives the coefficients $A_{i}$, $B_{i}, C_{i}$, and $D_{i}$ for Eqs. (5) and (6). Equations (5) and (6) reproduce the values from Refs. [3, 4] to better than $10^{-4} \%$ from $50 \mathrm{~K}$ to the triple-point temperature of $273.16 \mathrm{~K}$, although the lowest temperatures are not explored in this work.

Table 1. Coefficients for Eqs. (5) and (6).

\begin{tabular}{ccccc}
\hline$i$ & $A_{i}$ & $B_{i}$ & $C_{i}$ & \multicolumn{1}{c}{$D_{i}$} \\
\hline 1 & -1.88260766 & -1.83894213 & -3.40475823 & -2.99246875 \\
2 & 1.29472153 & 1.19552040 & 5.08793882 & 3.78091579 \\
3 & -0.11198310 & -0.03299456 & -3.81406793 & -2.15932630 \\
4 & 0.01315008 & - & 1.4096311 & 0.53566859 \\
5 & 0.00443206 & - & -0.10399425 & -0.00784145 \\
6 & - & - & - & 0.06090404 \\
\hline
\end{tabular}

For $v_{\mathrm{w}}^{\text {sat }}$ of liquid water, an accurate correlation has already been produced by Wagner and Pruss [7] and adopted as an IAPWS Supplementary Release [8]. In order to be consistent with the way IAPWS-95 is constructed, we use in Eqs. (3) and (4) its mass-based value of the gas constant $\left(R_{\mathrm{m}}=0.46151805\right.$ $\mathrm{kJ} \mathrm{kg}^{-1} \mathrm{~K}^{-1}$ [1]), and therefore also a mass-based specific volume that is the reciprocal of the liquid density at saturation given by Wagner and Pruss [7].

For $\kappa_{T}^{\text {sat }}$ of liquid water, values computed from IAPWS-95 were fitted to

$$
\kappa_{T}^{\text {sat,liq }}(T)=\kappa_{T, \mathrm{t}}^{\text {sat,liq }} \frac{1}{\sum_{j=1}^{8} N_{i} r^{t_{i}}},
$$

where the temperature variable $r$ is defined by $r=(T-225 \mathrm{~K}) /(10 \mathrm{~K})$, with the coefficients $N_{i}$ and exponents $t_{i}$ given in Table 2. The isothermal compressibility for liquid water at the triple point is $\kappa_{T, \mathrm{t}}^{\text {satliq }}$ $=5.089567 \times 10^{-10} \mathrm{~Pa}^{-1}$. Equation (7) reproduces $\kappa_{T}^{\text {sat,liq }}$ within $0.1 \%$ at temperatures from $235 \mathrm{~K}$ to $574 \mathrm{~K}$ (except for slightly larger errors below $242 \mathrm{~K}$ ), although only temperatures up to $473.15 \mathrm{~K}$ are explored here. Values below $273.16 \mathrm{~K}$ correspond to the supercooled liquid at vapor-liquid saturation. 
Table 2. Coefficients for Eq. (7).

\begin{tabular}{ccc}
\hline$i$ & $N_{i}$ & $t_{i}$ \\
\hline 1 & -4.7282 & -3.4 \\
2 & 9.3176 & -2.9 \\
3 & -10.158 & -1.9 \\
4 & 6.5439 & -1.3 \\
5 & -34.434 & 0.37 \\
6 & 34.2825 & 0.4 \\
7 & -0.709844 & 1.26 \\
8 & 0.337 & 1.4 \\
\hline
\end{tabular}

The evaluation of the Poynting correction also requires the saturation pressure as a function of temperature, $p^{\text {sat }}(T)$. For liquid water above the triple point, the correlation given by Wagner and Pruss $[7,8]$ is straightforward and is in excellent agreement with the full solution of IAPWS-95. A similar highaccuracy representation of $p^{\text {sat }}(T)$ for ice is the IAPWS formulation described by Wagner et al. [9, 10].

Finally, representations of $v_{\mathrm{w}}^{\text {sat }}(T), \kappa_{T}^{\text {sat }}(T)$, and $p^{\text {sat }}(T)$ are needed for supercooled liquid water. For $v_{\mathrm{w}}^{\mathrm{sat}}$, values at temperatures down to $235 \mathrm{~K}$ were generated from IAPWS-95 (note that this is an extrapolation below its official range of validity) and fitted to

$$
\frac{v_{\mathrm{w}}^{\text {sat,liq }}(T)}{v_{\mathrm{w}, \mathrm{t}}^{\mathrm{sat}, \mathrm{liq}}}=1+\frac{\exp \left[\left(\sum_{i=1}^{7} E_{i} s^{c_{i}}\right)^{\frac{1}{0.7}}\right]-1}{100},
$$

where the temperature variable is $s=\left(T_{\mathrm{t}}-T\right) /(10 \mathrm{~K})$ and the saturated liquid volume at the triple point is $v_{\mathrm{w}, \mathrm{t}}^{\text {sat liq }}=1.00020752 \times 10^{-3} \mathrm{~m}^{3} / \mathrm{kg}$. The coefficients $E_{i}$ and exponents $c_{i}$ are given in Table 3 . As done for liquid water above the triple point, a mass-based specific volume is used for supercooled water to match the mass-based gas constant of IAPWS-95 [1]. For $\kappa_{T}^{\text {sat }}(T)$, Eq. (7) is used. For $p^{\text {sat }}$, rather than extrapolate the correlation of Wagner and Pruss [7], we use the correlation of Murphy and Koop [11], because it was designed for this region. Note that, because $p^{\text {sat }}$ at these temperatures is small, the results for the supercooled liquid (and also for ice) are relatively insensitive to the vapor-pressure correlation used.

Table 3. Coefficients for Eq. (8).

\begin{tabular}{ccc}
\hline$i$ & $E_{i}$ & $c_{i}$ \\
\hline 1 & 0.1516 & 0.6995 \\
2 & 0.12856 & 1.654 \\
3 & 0.025921 & 3.7 \\
4 & -0.084443 & 4.2 \\
5 & 0.082210 & 4.7 \\
6 & -0.046209 & 5.3 \\
7 & 0.018246 & 5.55 \\
\hline
\end{tabular}

\section{Evaluation for Ice}

The first-order and second-order Poynting corrections for ice were evaluated at temperatures from $-100{ }^{\circ} \mathrm{C}$ to the triple point, at pressures up to $20 \mathrm{MPa}$. This range of conditions was chosen for its relevance to humidity metrology and to atmospheric and climate science. 


\section{Journal of Research of the National Institute of Standards and Technology}

At each condition, we first evaluated the Poynting correction $\Pi$ rigorously from the full thermodynamic potential for ice [3, 4], using Eq. (1). In order to illustrate the magnitude of the Poynting correction, in Fig. 1 we plot its values over the range considered. We then evaluated the first-order Poynting correction from Eq. (3) and the second-order correction from Eq. (4). The relative errors in the first-order and second-order approximations are $\left(\Pi_{1}-\Pi\right) / \Pi$ and $\left(\Pi_{2}-\Pi\right) / \Pi$, respectively.

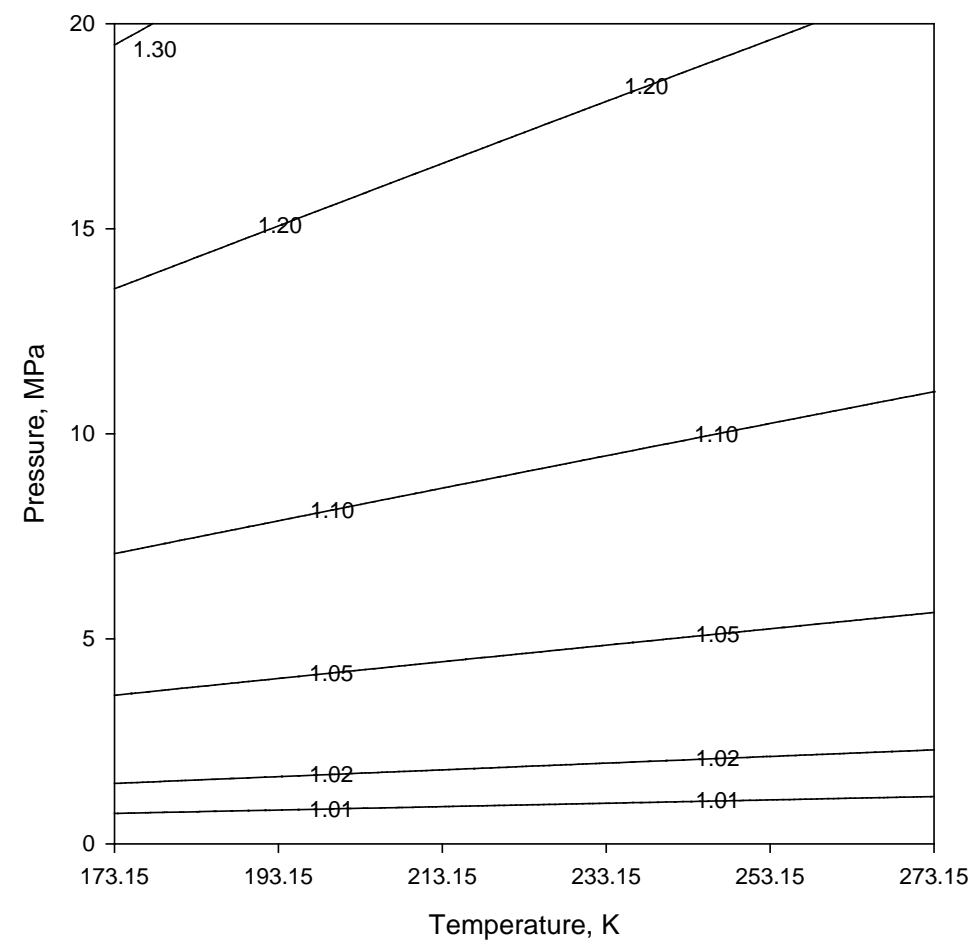

Fig. 1. Values of the Poynting correction for ice as a function of temperature and pressure.

In Fig. 2, we plot contours of the relative error in the first-order approximation multiplied by $10^{6}$ (i.e., parts per million). Since much metrology takes place at pressures near atmospheric, in Fig. 3 we restrict the maximum pressure to $1 \mathrm{MPa}$ in order to show more detail in this region. From Fig. 3, the error in the firstorder approximation for ice is quite small (less than $0.01 \times 10^{-6}$ ) at pressures near $0.1 \mathrm{MPa}$, and it is less than $0.7 \times 10^{-6}$ for the entire range of Fig. 3 . Figure 2 shows that the error grows significantly larger at high pressures. In both figures, we see that, for a fixed pressure, the error becomes slightly larger at lower temperatures. The fact that the error is positive at all conditions results from the decrease of $v_{\mathrm{w}}$ with pressure, so that assuming its value to be $v_{\mathrm{w}}^{\text {sat }}$ at all pressures overestimates the integral in Eq. (2). 


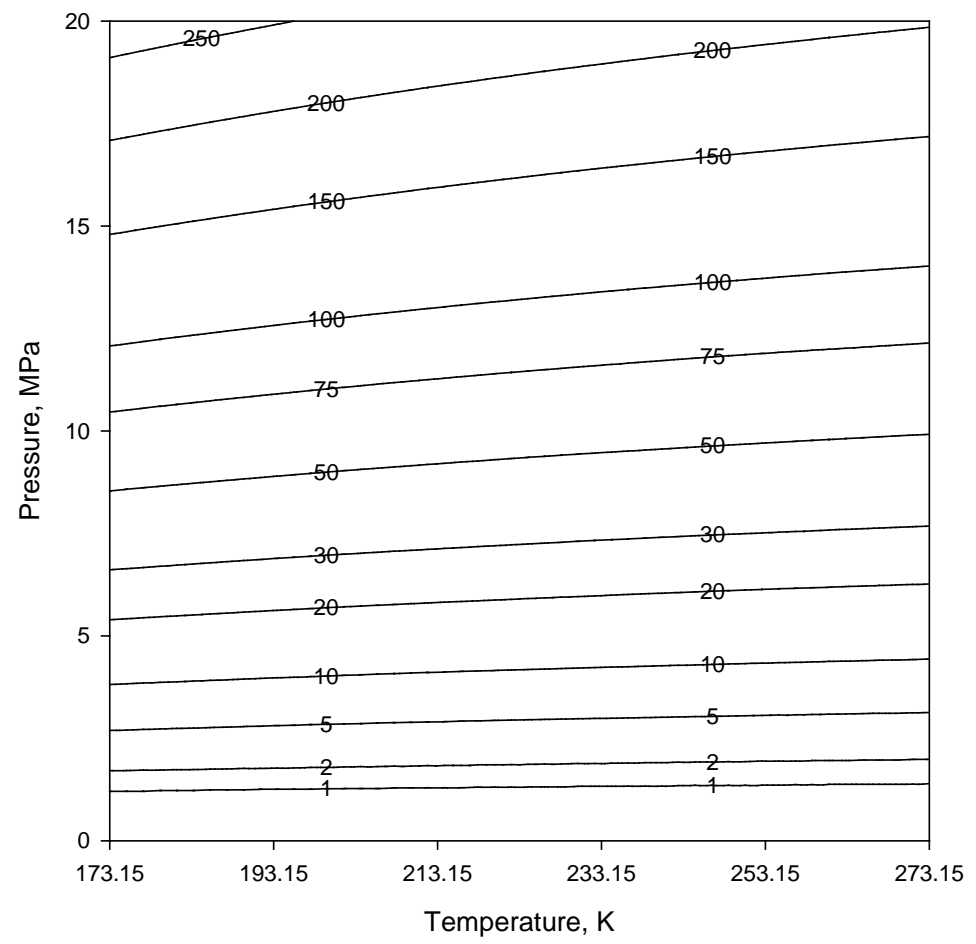

Fig. 2. Relative error of the first-order approximation to the Poynting correction, Eq. (3), for ice. Contours are in $10^{-6}$.

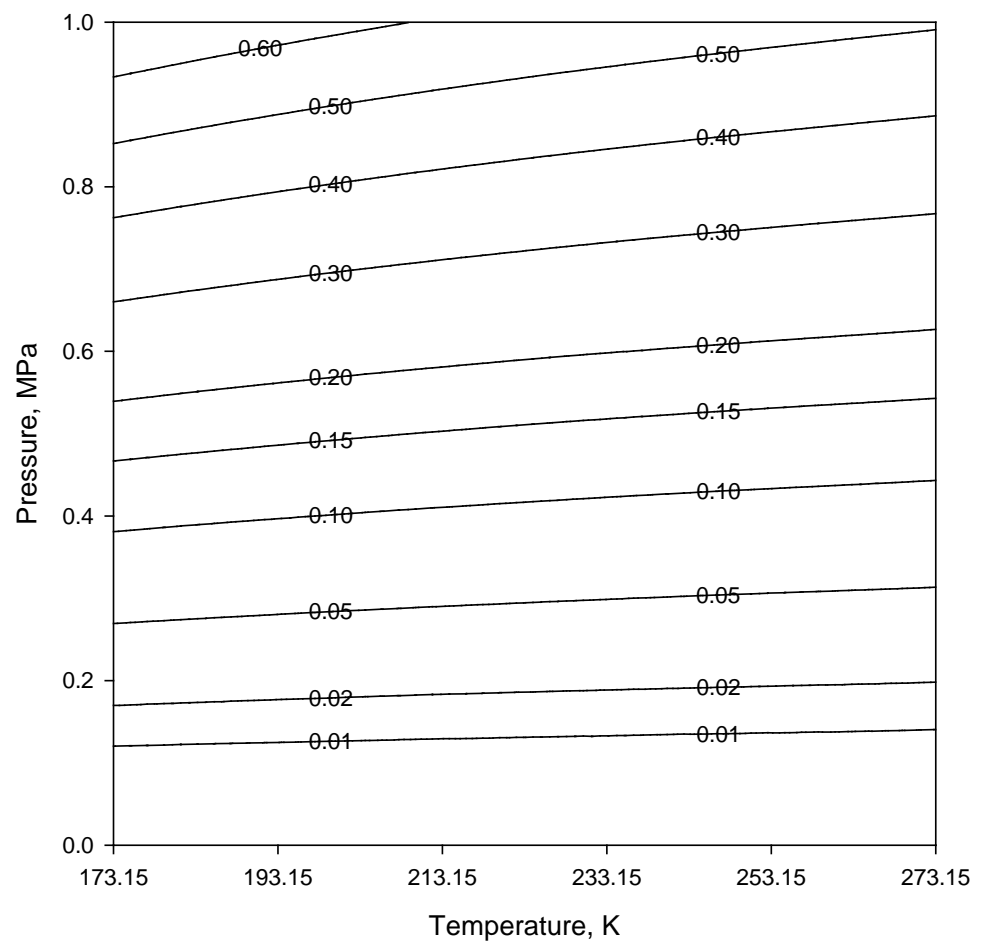

Fig. 3. Relative error of the first-order approximation to the Poynting correction, Eq. (3), for ice at pressures below 1 MPa. Contours are in $10^{-6}$. 
In Fig. 4, we similarly plot the relative error in the second-order approximation for ice. Figure 4 indicates that Eq. (4) produces excellent results throughout the range studied, with the magnitude of the error only reaching $1 \times 10^{-6}$ at the highest pressures and lowest temperatures. The second-order errors are negative, meaning that the Poynting correction is underestimated; this is because ice becomes less compressible with increasing pressure. A low-pressure plot for the second-order approximation is not shown because its relative error below $1 \mathrm{MPa}$ is tiny, with magnitude less than $0.0002 \times 10^{-6}$ for the entire region shown in Fig. 3.

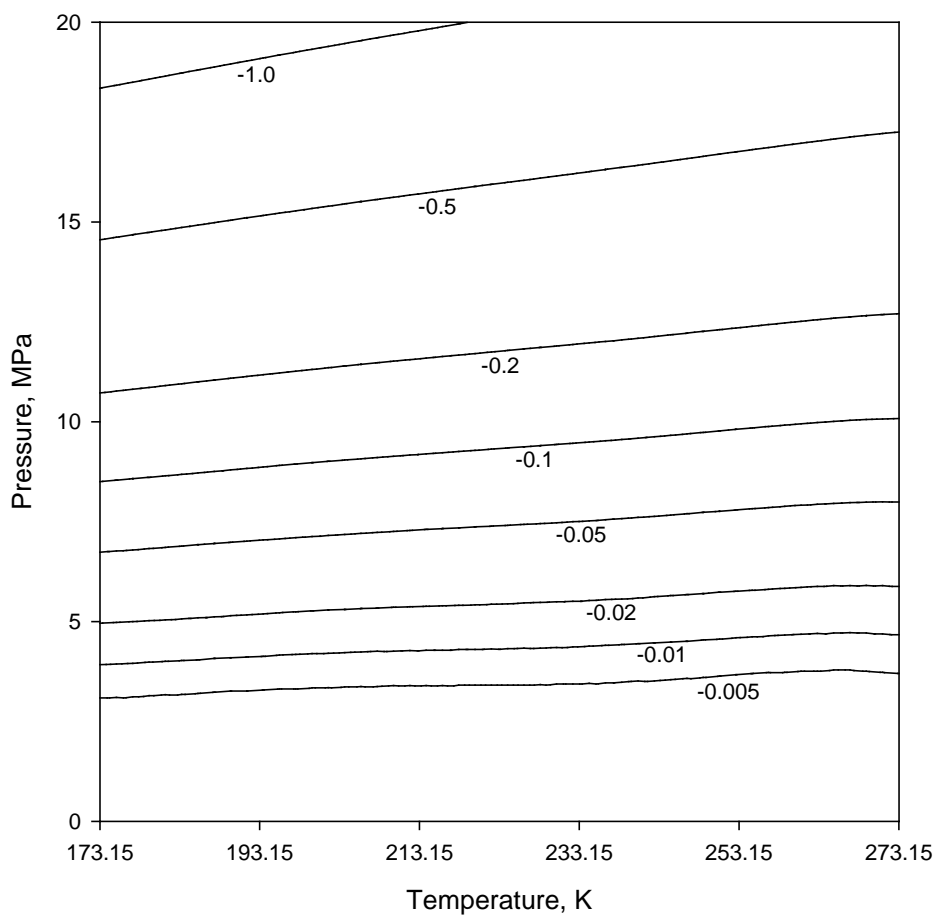

Fig. 4. Relative error of the second-order approximation to the Poynting correction, Eq. (4), for ice. Contours are in $10^{-6}$.

\section{Evaluation for Liquid Water}

The first-order and second-order approximations were evaluated for liquid water at temperatures from $0{ }^{\circ} \mathrm{C}$ to $200{ }^{\circ} \mathrm{C}$, again at pressures up to $20 \mathrm{MPa}$. Thermodynamic properties from the IAPWS-95 standard formulation [1, 2] were computed with a National Institute of Standards and Technology (NIST) Standard Reference Database [12]. Figure 5 shows a plot of the Poynting correction itself; the magnitude is similar to that shown for ice in Fig. 1.

Figure 6 shows the relative error in the first-order approximation over the entire range, while Fig. 7 shows the same quantity in more detail at pressures up to $1 \mathrm{MPa}$. The saturation curve $p^{\text {sat }}(T)$, where the Poynting correction is identically 1, is also plotted in Figs. 5-7. The behavior is similar to that shown in Figs. 2 and 3 for ice, except that the errors for liquid water at high pressures are more than twice as large. This is primarily due to the larger isothermal compressibility of liquid water compared to ice.

In Fig. 8, we plot the relative error in the second-order approximation for liquid water over the same range of conditions. While the errors are larger than they were for ice (see Fig. 4), they are still quite small, except at the highest pressures and temperatures studied, where they reach $-30 \times 10^{-6}$. These larger errors are due to water's isothermal compressibility changing more quickly with pressure at high temperatures. The region of very small positive errors in Fig. 8 is not physically significant; it results from imperfect representation of the IAPWS-95 saturated liquid volume by the correlation of Ref. [7]. We again do not present a low-pressure plot for the second-order approximation; its errors for pressures below $1 \mathrm{MPa}$ are less than $0.04 \times 10^{-6}$ for the entire region shown in Fig. 7 . 


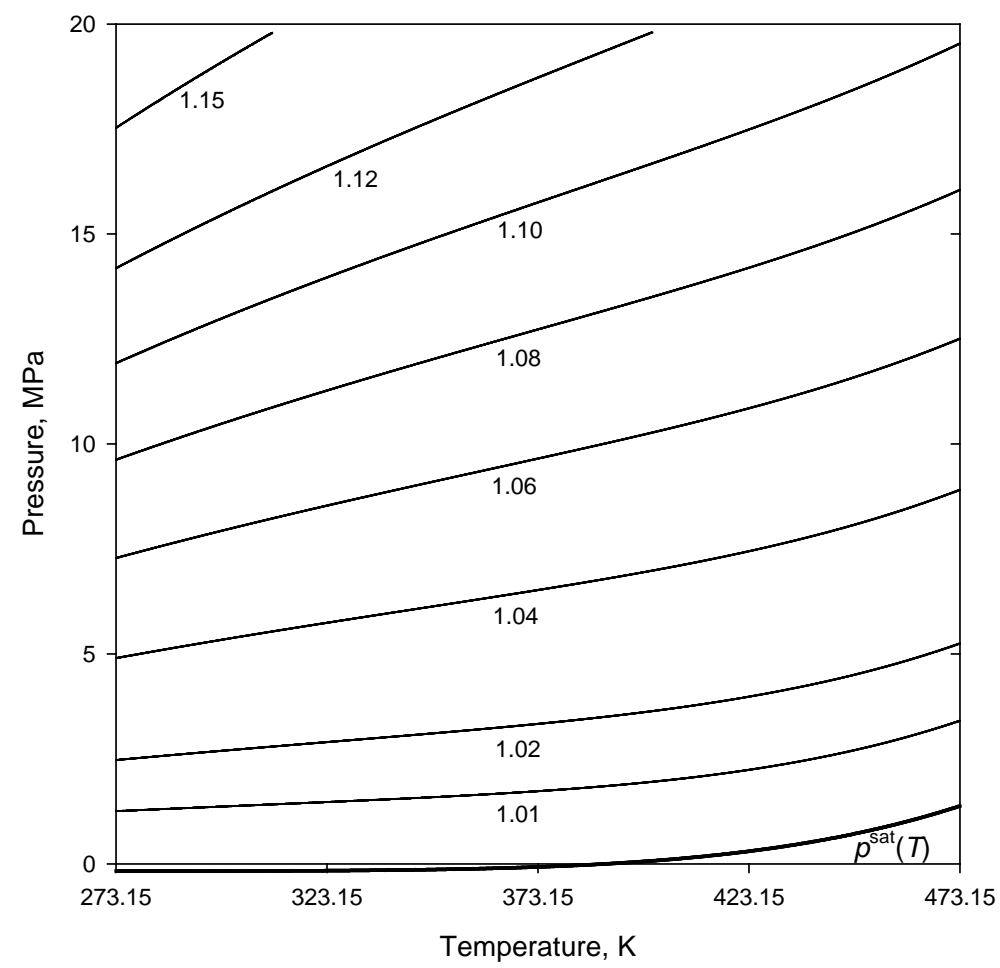

Fig. 5. Values of the Poynting correction for liquid water as a function of temperature and pressure.

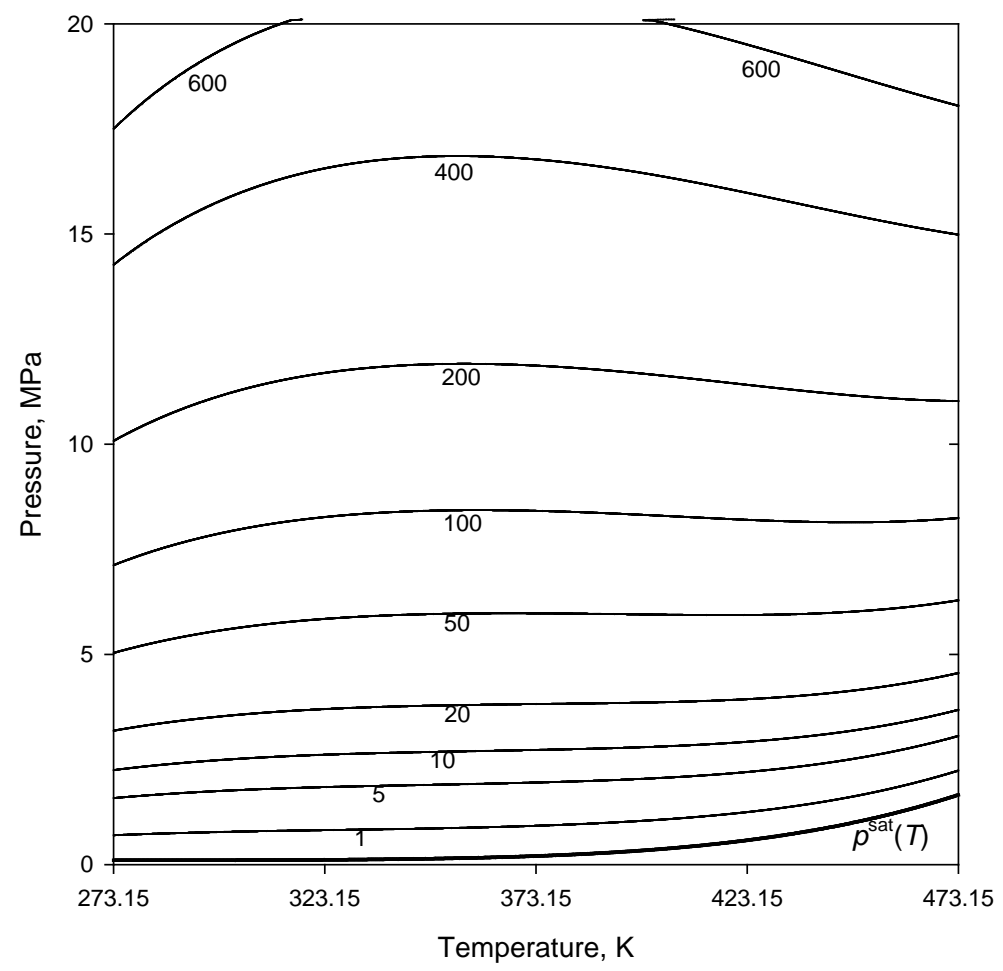

Fig. 6. Relative error of the first-order approximation to the Poynting correction, Eq. (3), for liquid water. Contours are in $10^{-6}$. 


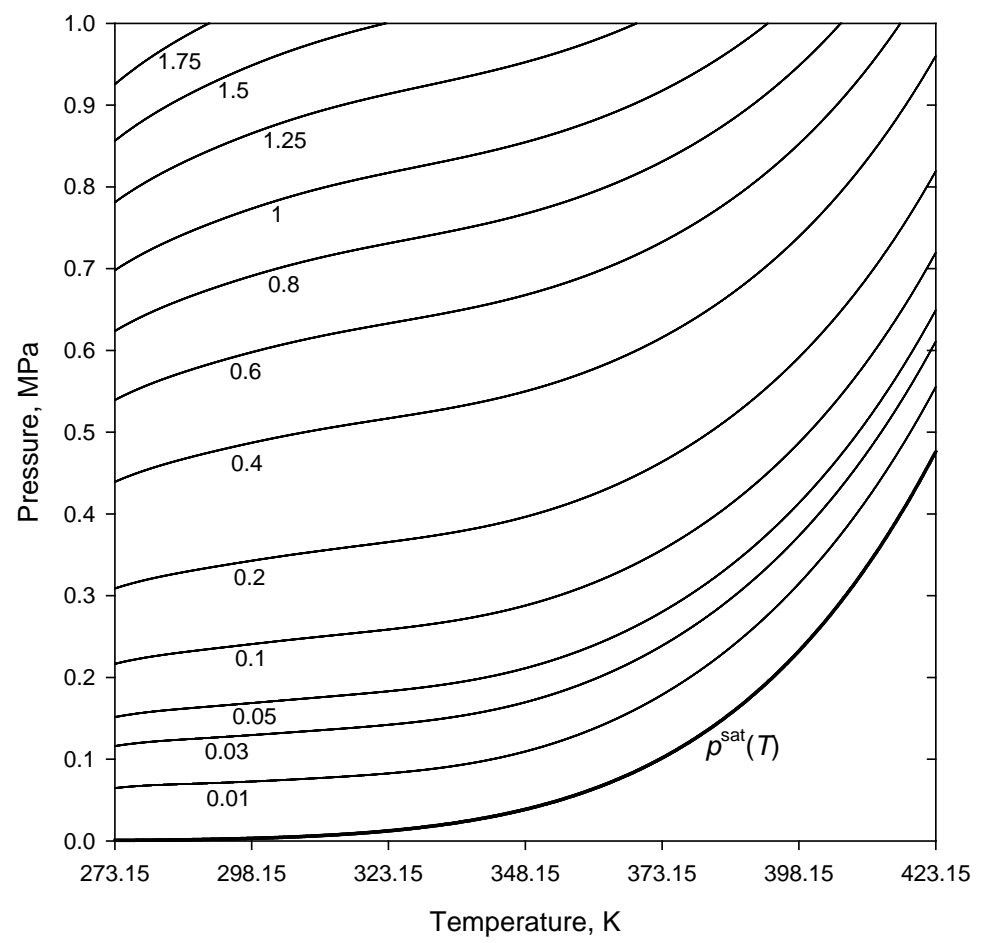

Fig. 7. Relative error of the first-order approximation to the Poynting correction, Eq. (3), for liquid water at pressures below $1 \mathrm{MPa}$. Contours are in $10^{-6}$.

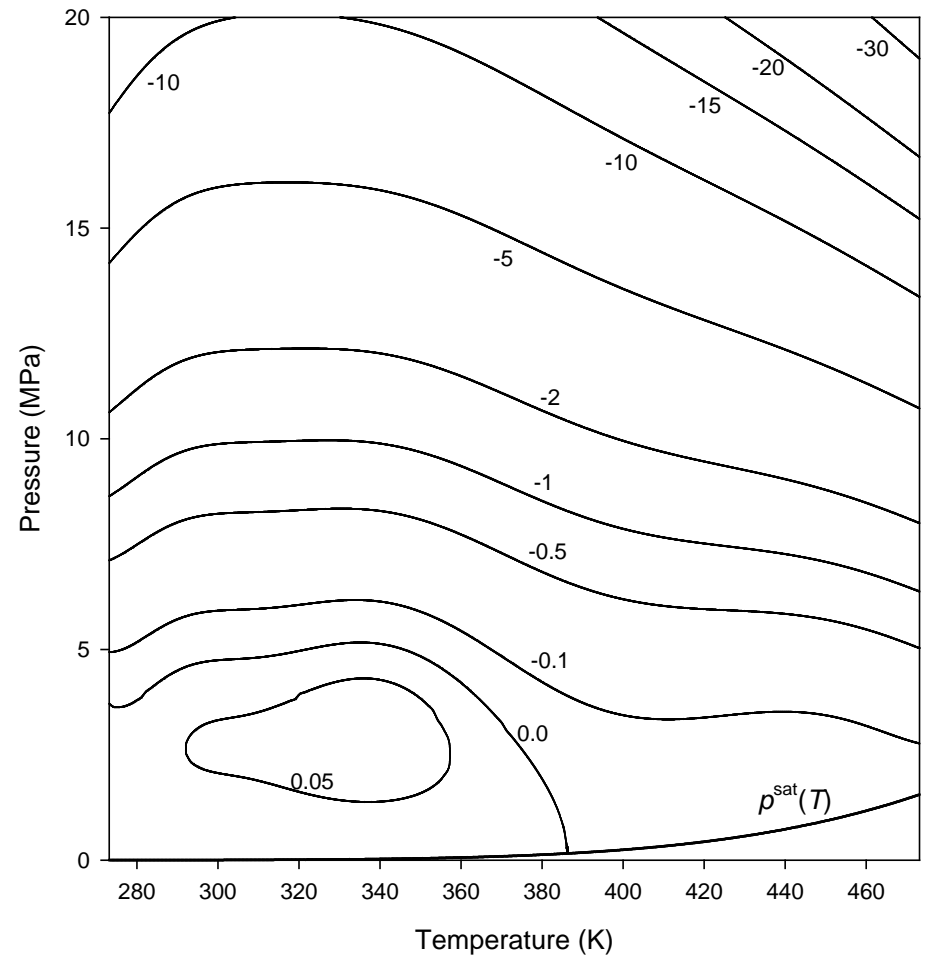

Fig. 8. Relative error of the second-order approximation to the Poynting correction, Eq. (4), for liquid water. Contours are in $10^{-6}$. 


\section{Evaluation for Supercooled Liquid Water}

It is common in the atmosphere for liquid water to exist as supercooled droplets, metastable with respect to an equilibrium ice phase. In humidity metrology, chilled-mirror instruments may produce coexistence of the vapor with a supercooled liquid rather than with ice. Therefore, it is valuable to examine the Poynting correction for these conditions. We examine conditions down to $235.15 \mathrm{~K}\left(-38{ }^{\circ} \mathrm{C}\right.$ ), which is not far above the lowest temperature at which metastable liquid can exist at atmospheric pressure [13]; this limit was also chosen because of unphysical ripples in the saturated volume behavior calculated from IAPWS-95 when it is extrapolated below about $238 \mathrm{~K}$. The upper pressure limit is again $20 \mathrm{MPa}$. Figure 9 shows the Poynting correction in this region; as expected, the values are similar to those for ice at the same conditions (Fig. 1).

In Fig. 10, we show the error in the first-order approximation, with Fig. 11 showing more detail for pressures below $1 \mathrm{MPa}$. As expected, the behavior is a continuation of Figs. 6 and 7 to lower temperatures. The error in the first-order approximation increases at lower temperatures, mainly due to the larger isothermal compressibility at these temperatures. The extrema in the contours near $236 \mathrm{~K}$ in Fig. 11 result from the aforementioned irregular behavior of IAPWS-95 when extrapolated to such low temperatures, and from the inability of Eq. (8) to perfectly reproduce that behavior.

In Fig. 12, we plot the relative error in the second-order approximation, Eq. (4), for supercooled liquid water. The errors are small, but not as small as in the cases of ice and stable liquid water (especially at the lowest temperatures). This is because the isothermal compressibility is larger and varies more strongly with pressure for supercooled water. The unusual structure at the lowest temperatures is again due to small deviations between Eq. (8) and the behavior of IAPWS-95 in this region. Note that the contours lowest in magnitude in Figs. 8 and 12 do not exactly match at 273.16 K; this is because Eq. (8) (used for Fig. 12) and the correlation of Ref. [7] (used for Fig. 8) do not quite give identical $v_{\mathrm{w}}^{\text {sat }}$ values at 273.16 K. We again do not give a low-pressure plot for the second-order approximation; the error of Eq. (4) for pressures below $1 \mathrm{MPa}$ is less than $1 \times 10^{-6}$ for the entire region shown in Fig. 11 (and only exceeds $0.3 \times 10^{-6}$ at temperatures below $238 \mathrm{~K}$ ).

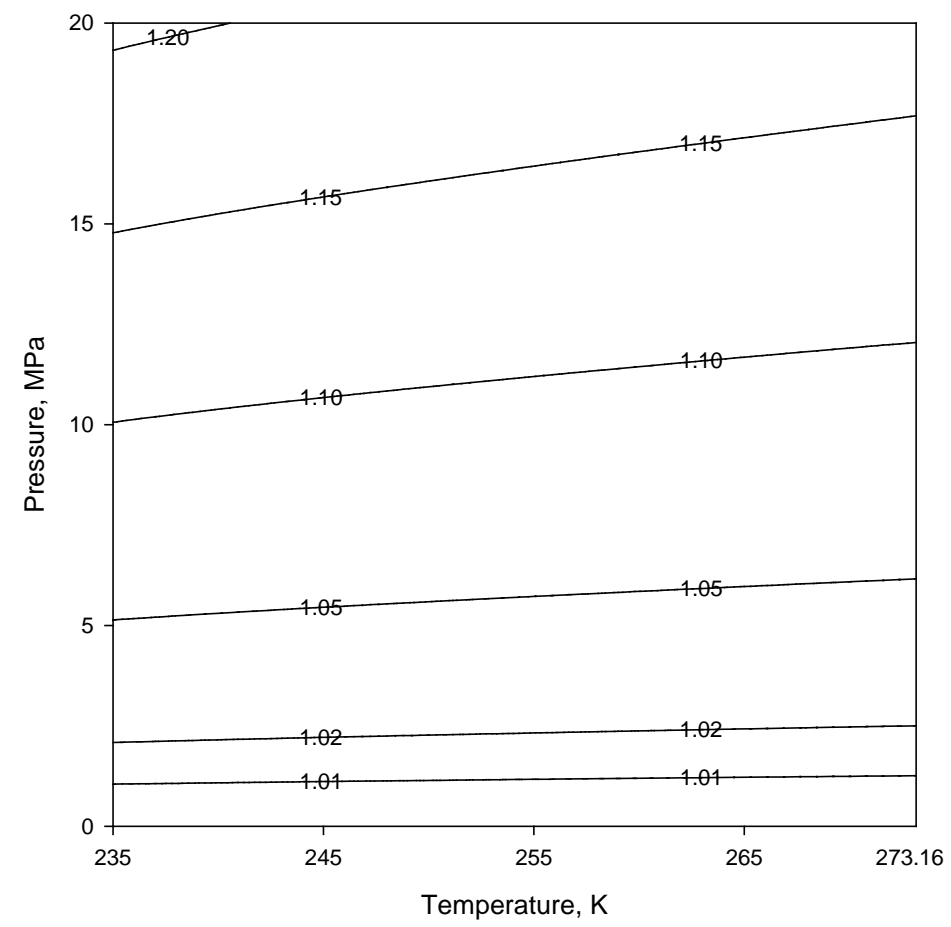

Fig. 9. Values of the Poynting correction for supercooled liquid water as a function of temperature and pressure. 


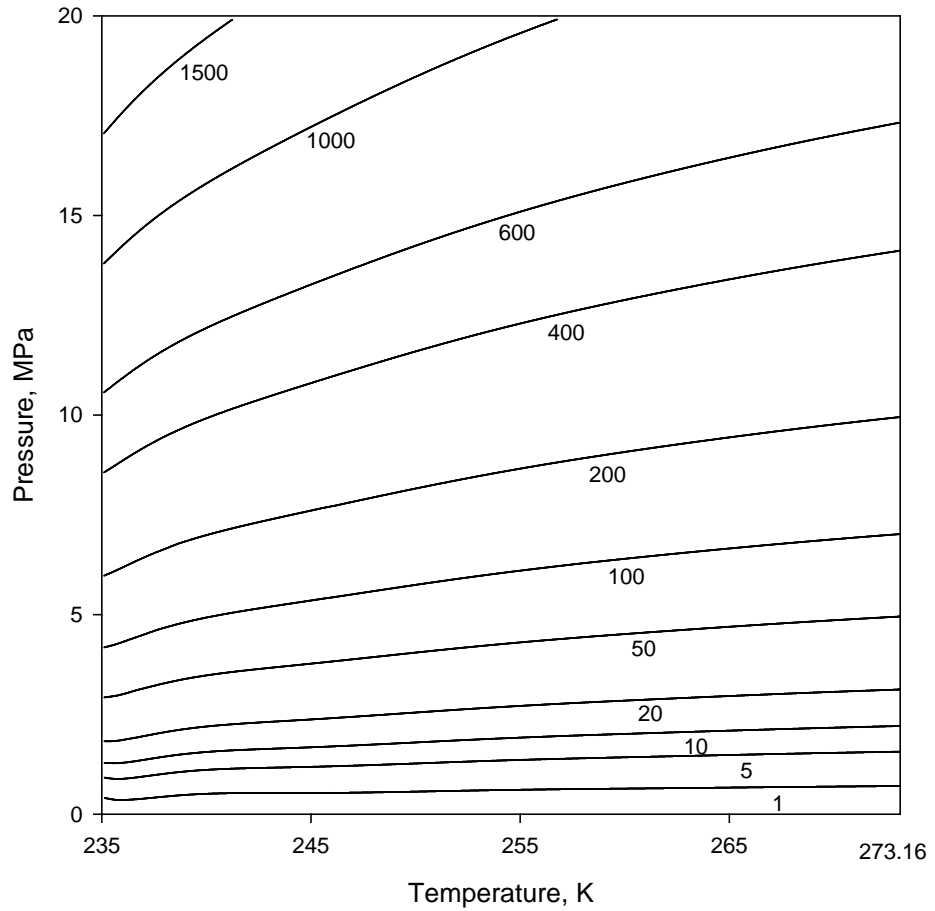

Fig. 10. Relative error of the first-order approximation to the Poynting correction, Eq. (3), for supercooled liquid water. Contours are in $10^{-6}$.

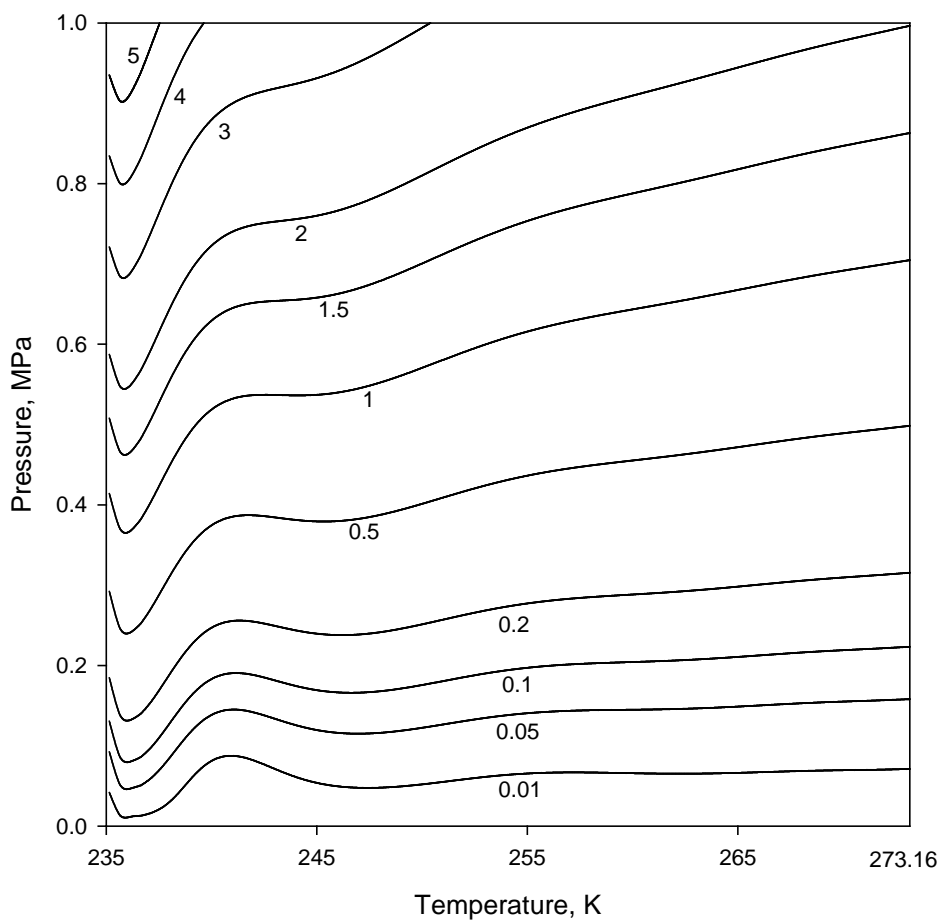

Fig. 11. Relative error of the first-order approximation to the Poynting correction, Eq. (3), for supercooled liquid water at pressures below $1 \mathrm{MPa}$. Contours are in $10^{-6}$. 


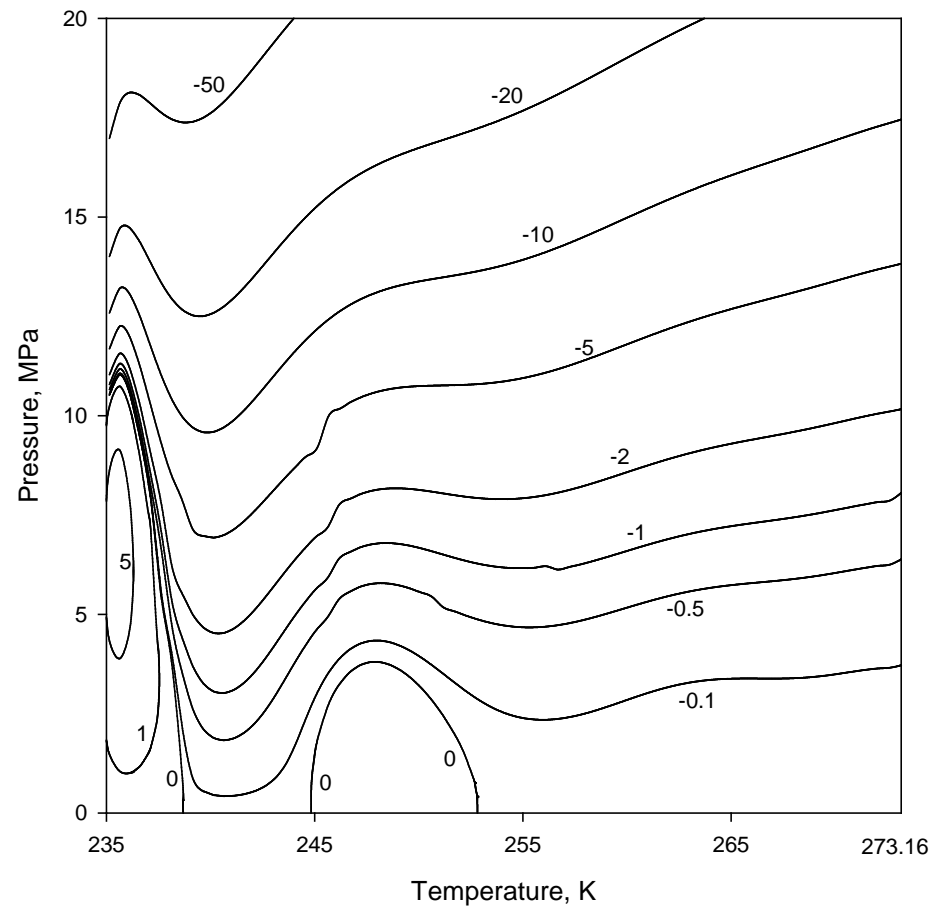

Fig. 12. Relative error of the second-order approximation to the Poynting correction, Eq. (4), for supercooled liquid water. Contours are in $10^{-6}$.

\section{Implications for Enhancement Factor Calculations}

In many problems, for example in humidity metrology, the actual interest is not in the Poynting correction itself, but rather in the enhancement factor, of which the Poynting correction is only one part. The enhancement factor $f$ is the ratio of the partial pressure of water in the vapor to the water saturation pressure at that temperature:

$$
f(T, p)=\frac{y_{\mathrm{w}} p}{p_{\mathrm{w}}^{\mathrm{sat}}(T)},
$$

where $y_{\mathrm{w}}$ is the equilibrium vapor-phase mole fraction of water. The enhancement factor measures the degree to which the amount of water in the vapor phase is "enhanced" due to the applied pressure (of air or another gas); the main contributors to this enhancement are the Poynting correction and the nonideality of the vapor phase.

If the solubility of the gas in water is small, equating the fugacities of water in the vapor and liquid produces a relatively simple expression for the enhancement factor [14]:

$$
f(T, p)=\frac{\left(1-x_{\mathrm{gas}}\right) \phi_{\mathrm{w}}^{\mathrm{sat}}(T) \Pi(T, p)}{\phi_{\mathrm{w}}^{\mathrm{vap}}(T, p, y)},
$$

where $x_{\text {gas }}$ is the mole fraction of dissolved gas in the condensed phase (which will be zero for ice and can be computed from Henry's law for gases in liquid water at low and moderate pressures), $\phi_{\mathrm{w}}^{\text {vap }}$ is the fugacity coefficient of water in the equilibrium vapor, and $\boldsymbol{y}$ is the vector of mole fractions representing the equilibrium composition of the vapor phase. 
Rigorous solution of Eq. (10) requires relatively complex calculations from the pure-water thermodynamic potential for $\Pi$, and from a mixture thermodynamic model for $\phi_{\mathrm{w}}^{\text {vap }}\left[\phi_{\mathrm{w}}^{\text {sat }}(T)\right.$ is a pure-water property that can be easily approximated]. Iteration is typically needed because $\phi_{\mathrm{w}}^{\text {vap }}$ and $x_{\text {gas }}$ depend on the unknown quantity $y_{\mathrm{w}}$.

Meaningful simplification of Eq. (10) must include approximations for both $\Pi$ and $\phi_{\mathrm{w}}^{\mathrm{vap}}$, and it is also desirable to minimize iteration. The work presented here will allow practitioners to evaluate the accuracy of approximations for $\Pi$; the second-order approximation should be adequate for almost all purposes, and at low pressures the first-order approximation is quite accurate. A straightforward expression of $\phi_{\mathrm{w}}^{\text {vap }}$ from the virial expansion (truncated at the level of third virial coefficients) was recently presented by Feistel et al. [15]; this expression should be accurate up to $5 \mathrm{MPa}$ and can also be used to compute $\phi_{\mathrm{w}}^{\text {sat }}$. The combination of this work and that of Feistel et al. [15] yields a relatively simple expression for $f$. It still requires iteration, but a good initial guess can be obtained by assuming an enhancement factor of 1 and using the resulting vapor composition to compute $\phi_{\mathrm{w}}^{\text {vap }}$ and $x_{\text {gas }}$.

\section{Conclusions}

The accuracies of both the common first-order approximation and a second-order approximation to the Poynting correction have been examined for condensed phases of ice, stable liquid water, and supercooled liquid water. The first-order approximation is Eq. (3), with the volume at saturation given by Eq. (5) for ice, by the correlation of Wagner and Pruss [7] for liquid water at $273.16 \mathrm{~K}$ and above, and by Eq. (8) for liquid water below $273.16 \mathrm{~K}$. The second-order approximation is given by Eq. (4), with the saturation volumes computed as in the first-order approximation and the isothermal compressibility at saturation given by Eq. (6) for ice and by Eq. (7) for liquid water.

Contour plots are given to illustrate the errors in both approximations as a function of temperature and pressure. At pressures below $1 \mathrm{MPa}$, the errors in the first-order approximation (Figs. 3, 7, and 11) are small (less than $0.7 \times 10^{-6}$ for ice, less than $2 \times 10^{-6}$ for liquid water, and less than $7 \times 10^{-6}$ for supercooled liquid water). The errors in the second-order approximation at such pressures are negligible (less than $0.0002 \times 10^{-6}$ for ice, less than $0.04 \times 10^{-6}$ for liquid water, and less than $1 \times 10^{-6}$ for supercooled liquid water). Results are displayed for higher pressures, up to $20 \mathrm{MPa}$, where the errors grow larger, especially for liquid water, where the first-order errors are more than twice those for ice at the same pressure. The errors of the second-order approximation remain quite small, especially for ice, as can be seen in Figs. 4, 8, and 12. The errors in the approximations are somewhat worse in general for supercooled liquid water, especially at very low temperatures where the isothermal compressibility becomes large.

In practice, it may be preferable to always use the second-order approximation, since it greatly reduces the error while retaining a relatively simple explicit form. The new correlations developed in this work for the volume and isothermal compressibility of ice at saturation, for the volume of supercooled liquid water at saturation, and for the saturated isothermal compressibility of liquid water (both stable and supercooled) enable straightforward calculation with these approximations.

While the figures in this paper have an upper pressure limit of 20 MPa, Eqs. (3) and (4) may be used at higher pressures and temperatures. In the Supplemental Material, we have supplied tables giving the Poynting correction and the errors of the first- and second-order approximations at pressures up to $100 \mathrm{MPa}$ and temperatures up to $573.15 \mathrm{~K}$. These tables may also be useful for finding a value of the error at a specific point for an uncertainty budget; such a value may be obtained by interpolation in the appropriate table. Interpolation may also be used to obtain values of the Poynting correction itself; such an approach is less convenient than the approximations evaluated here, but it could still be more convenient than implementing the full IAPWS thermodynamic potential for the calculation.

\section{Supplemental Materials}

- $\quad$ File ICE.txt contains comma-separated values of temperature (in K), pressure (in MPa), Poynting correction, relative error in first-order approximation to the Poynting correction (in $10^{-6}$ ), and 
relative error in second-order approximation to the Poynting correction (in $10^{-6}$ ). A grid is covered for ice from $173.15 \mathrm{~K}$ to $273.16 \mathrm{~K}$, with pressures up to $100 \mathrm{MPa}$.

- File LIQ.txt contains the same information for liquid water from $273.16 \mathrm{~K}$ to $573.15 \mathrm{~K}$, with pressures up to $100 \mathrm{MPa}$.

- File SUPER.txt contains the same information for supercooled liquid water from $235.15 \mathrm{~K}$ to 273.16 K, with pressures up to $100 \mathrm{MPa}$.

\section{Acknowledgments}

This work was inspired by discussions with Rainer Feistel, Olaf Hellmuth, and Jeremy Lovell-Smith. Ian Bell and Eric Lemmon of NIST produced the fits for Eqs. (5) to (8).

\section{References}

[1] International Association for the Properties of Water and Steam (2016) IAPWS R6-95(2016), Revised Release on the IAPWS Formulation 1995 for the Thermodynamic Properties of Ordinary Water Substance for General and Scientific Use. Available at http://www.iapws.org/relguide/IAPWS-95.html.

[2] Wagner W, Pruß A (2002) The IAPWS Formulation 1995 for the thermodynamic properties of ordinary water substance for general and scientific use. Journal of Physical and Chemical Reference Data 31(2):387-535. https://doi.org/10.1063/1.1461829.

[3] International Association for the Properties of Water and Steam (2009) IAPWS R10-06(2009), Revised Release on the Equation of State 2006 for $\mathrm{H}_{2} \mathrm{O}$ Ice Ih. Available at http://www.iapws.org/relguide/Ice-2009.html.

[4] Feistel R, Wagner W (2006) A new equation of state for $\mathrm{H}_{2} \mathrm{O}$ ice Ih. Journal of Physical and Chemical Reference Data 35(2):1021-1047. https://doi.org/10.1063/1.2183324.

[5] Lovell-Smith JW, Feistel R, Harvey AH, Hellmuth O, Bell SA, Heinonen M, Cooper JR (2016) Metrological challenges for measurements of key climatological observables. Part 4: atmospheric relative humidity. Metrologia 53(1):R40-R59. https://doi.org/10.1088/0026-1394/53/1/R40.

[6] Feistel R, Lovell-Smith JW (2017) Defining relative humidity in terms of water activity. Part 1: definition. Metrologia 54(4):566-576. https://doi.org/10.1088/1681-7575/aa7083.

[7] Wagner W, Pruss A (1993) International equations for the saturation properties of ordinary water substance, revised according to the International Temperature Scale of 1990. Journal of Physical and Chemical Reference Data 22(3):783-787. https://doi.org/10.1063/1.555926.

[8] International Association for the Properties of Water and Steam (1992) IAPWS SR1-86(1992), Revised Supplementary Release on Saturation Properties of Ordinary Water Substance. Available at http://www.iapws.org/relguide/Supp-sat.html.

[9] Wagner W, Riethmann R, Feistel R, Harvey AH (2011) New equations for the sublimation pressure and melting pressure of $\mathrm{H}_{2} \mathrm{O}$ ice Ih. J. Phys. Chem. Ref. Data 40(4):043103. https://doi.org/10.1063/1.3657937.

[10] International Association for the Properties of Water and Steam (2011) IAPWS R14-08(2011), Revised Release on the Pressure along the Melting and Sublimation Curves of Ordinary Water Substance. Available at http://www.iapws.org/relguide/MeltSub.html.

[11] Murphy DM, Koop T (2005) Review of the vapour pressures of ice and supercooled water for atmospheric applications. Quarterly Journal of the Royal Meteorological Society 131(608):1539-1565. https://doi.org/10.1256/qj.04.94.

[12] Harvey AH, Lemmon EW (2013) NIST Standard Reference Database 10: NIST/ASME Steam Properties, Version 3.0 (NIST, Gaithersburg, MD). Available at https://www.nist.gov/srd/nist-10.

[13] Kanno H, Speedy RJ, Angell, CA (1975) Supercooling of water to $-92^{\circ} \mathrm{C}$ under pressure. Science 189(4206):880-881. https://doi.org/10.1126/science.189.4206.880.

[14] Prausnitz JM, Lichtenthaler, RN, Gomes de Azevedo E (1999) Molecular Thermodynamics of Fluid-Phase Equilibria (Prentice Hall, Upper Saddle River, NJ), 3rd Ed.

[15] Feistel R, Lovell-Smith JW, Hellmuth O (2015) Virial approximation of the TEOS-10 equation for the fugacity of water in humid air. International Journal of Thermophysics 36(1):44-68. https://doi.org/10.1007/s10765-014-1784-0.

About the author: Allan H. Harvey is a chemical engineer in the Applied Chemicals and Materials Division at NIST, where he develops models for thermodynamics of pure fluids and mixtures, with an emphasis on aqueous systems and systems of importance for metrology.

The National Institute of Standards and Technology is an agency of the U.S. Department of Commerce. 